\title{
Identificação das classes de metabólitos secundários em extratos etanólicos foliares de Campomanesia adaman- tium, Dimorphandra mollis, Hymenaea stigonocarpa, Kielmeyera lathrophytum e Solanum lycocarpum
}

\author{
Identification of the class of secondary metabolites in the foliar ethanolic extracts of \\ Campomanesia adamantium, Dimorphandra mollis, Hymenaea stigonocarpa, \\ Kielmeyera lathrophytum andSolanum lycocarpum
}

\section{Antonio Carlos Pereira de Menezes Filho ${ }^{1}$ \\ Carlos Frederico de Souza Castro ${ }^{2}$}

\footnotetext{
1 Mestrando em Agroquímica pelo Instituto Federal de Educação, Ciência e Tecnologia Goiano, Campus Rio Verde, GO, Brasil. Graduado em Ciências Biológicas pela Universidade de Rio Verde, GO, Brasil.

E-mail: astronomoamadorgoias@gmail.com 1 http://lattes.cnpq.br/1071427974283935 ${ }^{10}$ http://orcid.org/0000-0003-3443-4205

2 Doutor em Química pela Universidade de Brasília. Professor no Mestrado em Agroquímica pelo Instituto Federal de Educação, Ciência e Tecnologia Goiano, Campus Rio Verde, GO, Brasil.

E-mail: carlos.castro@ifgoiano.edu.br $\underline{\text { http://lattes.cnpq.br/6519321142404132 (iD http://orcid.org/0000-0002-9273-7266 }}$
}

\begin{abstract}
RESUMO: O bioma Cerrado apresenta inúmeras espécies vegetais que ainda possuem poucos dados na literatura a respeito dos compostos fitoquímicos. O objetivo deste estudo foi identificar através da triagem fitoquímica dos compostos químicos nos extratos foliares de C. adamantium, $D$. mollis, H. stigonocarpa, K. lathrophytum e S. lycocarpum. Folhas foram coletadas em uma área de Cerrado, onde foram produzidos extratos etanólicos sendo avaliados por testes fitoquímicos. Foram obtidos a partir dos testes fitoquímicos qualitativos a presença de compostos, de ácidos orgânicos, açúcares redutores, alcaloides, catequinas, cumarinas, depsídeos, depsidonas, ligações olefínicas, fenóis, flavonóides, glicosídeos cardiotônicos, saponinas e taninos, e não foram detectados compostos polissacarídicos e purinas nos cinco extratos etanólicos foliares avaliados. A pesquisa preliminar realizada para os extratos foliares avaliados possibilitou o conhecimento prévio indicando a natureza das substâncias presentes nos preparados populares.

Palavras-chave: Propriedades fitoquímicas, folhas, extração.
\end{abstract}

\begin{abstract}
The Cerrado biome presents numerous plant species that still have few data in the literature regarding the phytochemical compounds. The objective of this study was to identify the phytochemical chemical compounds in the leaf extracts of $C$. adamantium, D. mollis, H. stigonocarpa, K. lathrophytum and S. lycocarpum. Leaves were collected in a Cerrado area, where ethanolic extracts were produced and evaluated by phytochemical tests. The presence of compounds, organic acids, reducing sugars, alkaloids, catechins, coumarins, depesides, depsidones, olefinic bonds, phenols, flavonoids, cardiotonic glycosides, saponins and tannins were obtained from qualitative phytochemical tests and no polysaccharide compounds and purines in the five foliar ethanol extracts evaluated. The preliminary research carried out for the leaf extracts evaluated allowed previous knowledge indicating the nature of the substances present in the popular preparations.

Keywords: Phytochemical properties, leaves, extraction.
\end{abstract}

\section{INTRODUÇÃO}

O Cerrado é o segundo maior bioma natural ocupando uma área de quase 2 milhões de $\mathrm{km} 2$, cerca de $22 \%$ do território nacional, sendo considerado um bioma de transição entre todos os outros biomas brasileiros (BUENO et al., 2018). O Cerrado apresenta diversas fitofisionomias com grande variedade de espécies vegetais, atualmente estão identifi- 
cadas mais de 11.000 espécies de flora nativa, onde destas 4.400 são endêmicas, várias espécies são produtoras de frutos que alimentam a fauna local (MENDONÇA et al., 2008; MYERS et al., 2000).

Com essa pluralidade vegetal e aos compostos fitoquímicos, muitas espécies dessa flora são utilizadas como plantas medicinais aplicadas a terapias alternativas que a cada ano vem atingindo um público maior influenciado pela questão econômica, regiões longínquas com acesso quase restrito pela distância a uma unidade de saúde e ao difícil acesso ao Sistema único de Saúde (SUS). Isso requer maior empenho da pesquisa para fornecimento de informações sobre os compostos orgânicos majoritários (SILVA; OLIVEIRA; LIMA, 2015; LOLA-LUZ; HENNEQUART; GAFFNEY, 2014).

Para tanto, as análises fitoquímicas se fazem necessário para que se possa identificar os vários tipos de compostos em um mesmo extrato vegetal (raízes, caule, cascas, galhos, folhas, inflorescência e sementes). $\mathrm{O}$ isolamento de compostos orgânicos com características terapêuticas aplicadas a novos fitofármacos vem ganhando força no mercado de produtos naturais.

Para Silva, Miranda e Conceição (2010), a pesquisa fitoquímica te como pilar, conhecer os constituintes químicos que as plantas produzem como metabólitos secundários, muitos deles como agentes desencadeados por stress químico, biológico e físico, como, tipo de solo, sazonalidade, irradiação solar, pluviosidade e até queimadas. Os autores complementam que a necessidade de novos estudos abordando uma vasta quantidade de espécies da flora que ainda não possuem relatos dos seus compostos químicos conhecidos, torna-se a análise fitoquímica de extrema necessidade para identificação dos grupos de metabólitos secundários com características relevantes no cenário fitoterapêutico.
Os estudos fitoquímicos possuem grande vertente, visto que, não aborda apenas plantas medicinais conhecidas, mas sim o vasto banco vegetal natural como, por exemplo, a flora do Cerrado que apresenta uma alta variabilidade de espécies para obtenção de compostos bioativos, no desenvolvimento de novos alimentos funcionais e como fitoterápicos aplicados a inúmeras patologias que acometem humanos e animais (MELLO; SANTOS, 2001).

O estudo preliminar fitoquímico das espécies semi-arbustivas e arbustivas do Cerrado como a gabiroba (C. adamantium Berg.), faveiro (D. mollis Benth.), jatobá-do-cerrado ( $H$. stigonocarpa Mart. ex Hayne), pau-santo ( $K$. lathrophytum Saddi.) e lobeira ( $S$. lycocarpum A. St. - Hill.), torna-se necessário para que possamos compreender as informações fitoquímicas dos metabólitos secundários produzidos por essas espécies, com tudo alguns testes simples e de baixo custo podem ser realizados para a identificação dos principais compostos dos grupos dos flavonóides, alcalóides, taninos, cumarínicos, glicosídeos cardiotônicos e purinas apresentando atividades potencialmente benéficas para tratamento de inúmeras complicações médicas (SILVA; MIRANDA; CONCEIÇÃ̃, 2010).

O presente trabalho teve por objetivo realizar análises fitoquímicas preliminares a nível qualitativo em cinco espécies botânicas que coabitam no Cerrado tipo cerradão, pesquisando a presença de ácidos orgânicos, açúcares redutores, alcalóides, catequinas, cumarinas, depsídeos, depsidonas, duplas ligações olefínicas, fenóis, flavonóides, glicosídeos cardiotônicos, polissacarídeos, purinas, saponinas e taninos.

\section{MATERIAIS E MÉTODO}

\section{Material vegetal}

As folhas das espécies vegetais, Campomanesia adamantium Berg., Dimorphandra mollis Benth., Hymenaea stigonocarpa Mart. ex Hayne, 
Kielmeyera lathrophytum Saddi. eSolanum lycocarpum A. St. -Hill., foram coletadas em uma área de Cerrado pertencente à Universidade de Rio Verde-GO. A coleta foi realizada nas primeiras horas da manhã, as folhas de cada espécie vegetal foram armazenadas em embalagens plásticas de polietileno transparente $\mathrm{e}$ levadas para o laboratório de Química Tecnológica no Instituto Federal Goiano, Campus Rio Verde-GO.

\section{Preparo dos extratos etanólicos}

O preparo do extrato etanólico foi realizado conforme Menezes Filho e Castro (2019) com modificações. As folhas foram lavadas em água corrente e deixadas para retirada do excesso de água sob folhas de papel toalha. Logo após, as folhas foram trituradas em liquidificador doméstico até obtenção de um triturado homogêneo. Foram pesadas $100 \mathrm{~g}$ de material foliar triturado e logo em seguida foi realizada a extração utilizando álcool etílico $95 \%$.

A extração procedeu-se por 7 dias em local ao abrigo de luz e calor. Após esse tempo, as soluções foram filtradas em papel filtro qualitativo e o sobrenadante foi centrifugado em tubos Falcon de $50 \mathrm{~mL}$ a $3000 \mathrm{rpm}$ por 15 minutos. O sobrenadante após centrifugação, foi armazenado em frascos de cor âmbar e levados para geladeira a $8 \pm 1,0{ }^{\circ} \mathrm{C}$ até análises.

\section{Teste de determinação qualitativa para ácidos orgânicos}

Para determinação qualitativa de ácidos orgânicos seguiu conforme descrito por Gomes, Martins e Almeida (2017) com modificações. Foram utilizados $3 \mathrm{~mL}$ do extrato etanólico foliar dissolvidos em $5 \mathrm{~mL}$ de água destilada. Logo após, a amostra foi filtrada em papel filtro e $2 \mathrm{~mL}$ do sobrenadante foi adicionado em um tubo de ensaio acrescido com 5 gotas do reativo de Pascová. A descoloração do reativo indica a presença positiva de ácidos orgânicos na amostra.

\section{Teste para açúcares redutores}

Para análise qualitativa de açúcares redutores (AR), seguiu conforme proposto por Gomes, Martins e Almeida (2017) com modificações. Uma alíquota de $3 \mathrm{~mL}$ do extrato etanólico foliar foi acrescido com $5 \mathrm{~mL}$ de água destilada. Logo em seguida foram adicionados $2 \mathrm{~mL}$ de solução de Felhing A e $2 \mathrm{~mL}$ de solução Felhing B, posteriormente foi aquecido em banho-maria a $100 \pm 1,0{ }^{\circ} \mathrm{C}$ por 5 minutos. A presença de precipitado com coloração vermelho tijolo indica a presença de açúcares reduzidos.

\section{Testes qualitativos para alcaloides}

Para análise de alcaloides, seguiu conforme descrito por Menezes Filho e Castro (2019) e Barbosa et al. (2008) com modificações. Onde $2 \mathrm{~mL}$ de extrato foliar etanólico foi acrescido com $3 \mathrm{~mL}$ de uma solução aquosa de $\mathrm{HCl}$ $10 \%(\mathrm{~m} / \mathrm{v})$, sendo aquecido por 10 minutos a $100 \pm 1,0{ }^{\circ} \mathrm{C}$ em banho-maria. Em seguida, a solução foi esfriada a temperatura ambiente de $25 \pm 1,0^{\circ} \mathrm{C}$. Logo após, a solução foi separada em igual quantidade em dois tubos de ensaios.

No tubo 1, foi realizado utilizando 5 gotas do reativo de Mayer (1,36 g de $\mathrm{HgCl}_{2}$ em 60 $\mathrm{mL}$ de água e $5 \mathrm{~g}$ de $\mathrm{KI}$ em $10 \mathrm{~mL}$ de água destilada para $100 \mathrm{~mL}$ de solução) e no tubo 2 , realizou-se a reação com reativo de Wagner (1,27 g de $\mathrm{I}_{2}$ e $2 \mathrm{~g}$ de KI diluído em $5 \mathrm{~mL}$ de água destilada, completando-se para $100 \mathrm{~mL}$ ). Homogeneizando-se manualmente por $1 \mathrm{mi}-$ nuto.

Uma leve turbidez ou precipitado se forma no fundo do tubo, apresentando coloração (roxa a alaranjada, ao branco, creme e marrom) evidenciando a presença de metabólitos secundários. 


\section{Testes qualitativos para glicosí- deos cardiotônicos}

Para determinar a presença de alcaloides nos extratos etanólicos foliares, seguiu conforme descrito por Gomes, Martins e Almeida (2017) com modificações. Uma alíquota de 5 $\mathrm{mL}$ de extrato etanólico foliar foi acrescido com $5 \mathrm{~mL}$ de $\mathrm{HCl}$ 5\% (m/v). A solução preparada foi filtrada em papel filtro. A solução foi dividida em 4 tubos em igual partes, sendo cada tubo acrescidos com $1 \mathrm{~mL}$ da solução preparada.

No tubo 1, foi adicionado $1 \mathrm{~mL}$ do reagente de Baljet composto por (8 gotas de ácido acético P.A., e $3 \mathrm{~mL}$ de clorofórmio P.A.). A coloração se torna roxa, laranja-roxeada ou violeta, indicando a presença de glucosídeos cardiotônicos.

No tubo 2, foi adicionado $1 \mathrm{~mL}$ do reativo de Kedde (Solução A composta por ácido 3,5dinitrobenzóico a $3 \%$ em $10 \mathrm{~mL}$ metanol P.A., e Sol. B composta por uma solução aquosa de $\mathrm{KOH}$ a 5,7\%), foi adicionado. A coloração reagente apresenta tons de rosa ao azul-violeta indicando a presença de cardenólidos. Conforme Silva e Lima (2016) os compostos bufadienólidos não reagem.

Tubo 3, foi realizado a reação de KellerKilliani o reagente é composto por $(1 \mathrm{~mL}$ de ácido Acético P.A., duas gotas de cloreto férrico III a 5\% em metanol $(\mathrm{m} / \mathrm{v})$ e $1 \mathrm{~mL}$ de ácido Sulfúrico P.A.). Há formação do um anel na cor vermelho acastanhado, reação mais cor da fase acética azul esverdeada.

Tubo 4, foi realizado a reação de Raymond-Marthoud. O extrato depois de filtrado, foi adicionado 2 gotas de solução de cloreto férrico III a 10\% em metanol P.A., e duas gotas de acetato de chumbo a 10\%. A solução foi homogeneizada por 1 minuto em Vortex. O resultado positivo apresenta coloração amarela a roxo.

\section{Testes para catequinas}

Para determinação qualitativa de catequinas nos extratos etanólicos foliares, seguiu conforme descrito por Gomes, Martins e Almeida (2017) com modificações.

Teste A. Uma alíquota de $3 \mathrm{~mL}$ do extrato etanólico foliar, foi acrescido com $3 \mathrm{~mL}$ de metanol P.A., um palito de fósforo foi embebedado com esta solução e deixado para evaporar em temperatura ambiente de $25 \pm 1,0$ ${ }^{\circ} \mathrm{C}$ até ficar seco. Logo em seguida o palito foi umedecido em HCl P.A., e levado para uma chama de bico de Bunsen. A coloração vermelha da chama indica a presença de catequinas.

Teste B. Uma alíquota de $3 \mathrm{~mL}$ do extrato etanólico foliar foi acrescido com $1 \mathrm{~mL}$ de solução aquosa de vanilina $1 \%$ e mais $1 \mathrm{~mL}$ de $\mathrm{HCl}$ P.A. Ocorre mudança de coloração com formação de precipitado.

\section{Testes para fenóis, taninos e du- plas ligações olefínicas}

Para determinação qualitativa de fenóis e taninos seguiu conforme descrito por Gomes, Martins e Almeida (2017) com modificações. Uma alíquota de $3 \mathrm{~mL}$ de extrato etanólico foliar foi adicionado 3 gotas de uma solução etanólica de $\mathrm{FeCl}_{3}$ 1\% (m/v) e homogeneizado em Vortex por 30 segundos. A presença inicial de coloração entre o azul e vermelho indica a presença de fenóis.

A presença de precipitados escuros de tonalidade azul indica presença de taninos pirogálicos e em verde taninos catéquicos (BRITO et al., 2008).

Para duplas ligações olefínicas seguiu conforme descrito por De Ugaz e Lock (1994) com modificações. Foram utilizados $3 \mathrm{~mL}$ do extrato foliar etanólico, acrescidos com $2 \mathrm{~mL}$ de água destilada e $3 \mathrm{~mL}$ de uma solução aquosa de permanganato de potássio 0,01\% $(\mathrm{m} / \mathrm{v})$. A solução foi homogeneizada por 10 segundos em Vortex. Logo em seguida foram adicionadas 2 gotas de uma solução aquosa de 
$\mathrm{NaCO}_{3}$ 7,5\% (m/v) e homogeneizado novamente por mais 10 segundos em Vortex. A descoloração indica presença de duplas ligações olefínicas.

\section{Teste para flavonoides}

Para a determinação qualitativa de flavonoides (reação de Shinoda) seguiu conforme descrito por Gomes, Martins e Almeida (2017) com modificações. Uma alíquota de 5 $\mathrm{mL}$ do extrato etanólico foliar, foi acrescida com 15 gotas de $\mathrm{HCl} \mathrm{P.} \mathrm{A.,} \mathrm{foi} \mathrm{adicionado}$ raspas de magnésio e deixado sob descanso por 5 minutos. A presença de coloração rósea indica flavonoides no extrato.

\section{Teste qualitativo para polissacarí- deo}

O teste para polissacarídeos seguiu conforme descrito por Gomes, Martins e Almeida (2017) com modificações. Em um tubo de ensaio, foi adicionado $5 \mathrm{~mL}$ do extrato etanólico foliar e acrescido com 3 gotas de reagente de lugol. A coloração azul indica a presença de cadeias polissacarídicas nos extratos.

\section{Teste de qualitativo para purinas}

Para determinação de purinas seguiu conforme proposto por Gomes, Martins e Almeida (2017) com pequenas modificações. Em uma cápsula de porcelana, foi adicionado $3 \mathrm{~mL}$ do extrato etanólico foliar, com 3 gotas de uma solução aquosa de $\mathrm{HCl} 2 \mathrm{~N}(\mathrm{~m} / \mathrm{v})$ e duas gotas de $\mathrm{H}_{2} \mathrm{O}_{2} 30 \%$ (v/v). A solução foi levada para evaporação em estufa com circulação e renovação de ar forçada a $90 \pm 1,0{ }^{\circ} \mathrm{C}$. Após este processo, a formação de coloração avermelhada como resíduo foi acrescido com 3 gotas de uma solução aquosa de $\mathrm{NH}_{4} \mathrm{OH}$ $6 \mathrm{~N}(\mathrm{~m} / \mathrm{v})$. A coloração em tom violeta indica a presença positiva para purinas.

\section{Teste para derivados cumarínicos}

Para determinação qualitativa de cumarinas seguiu conforme proposto por Silva e Lima
(2016) com modificações. Uma alíquota de 5 $\mathrm{mL}$ do extrato etanólico foliar, foi aquecido em banho-maria a $90 \pm 1,0{ }^{\circ} \mathrm{C}$ até concentração de 0,5 mL. Em uma fita de $5 \times 5 \mathrm{~cm}$ foram pingadas 3 gotas do extrato concentrado, onde formou-se duas manchas com aproximadamente $1 \mathrm{~cm}$ cada.

A mancha 1 foi o controle e a mancha 2 foi adicionado 1 gota de solução de $\mathrm{NaOH}$ 10\% $(\mathrm{m} / \mathrm{v})$. A fita foi levada para câmara com luz ultravioleta e observada em dois comprimentos de ondas em 254 e $365 \mathrm{~nm}$. A presença de coloração amarela ou verde indica compostos cumarínicos no extrato.

\section{Teste qualitativo para saponinas}

A determinação da presença de saponinas seguiu conforme proposto por Kloss et al. (2016) com modificações. Uma alíquota de 2 $\mathrm{mL}$ de extrato etanólico foliar foi adicionado com $5 \mathrm{~mL}$ de água destilada fervente. A solução foi deixada para esfriar até temperatura de $25{ }^{\circ} \mathrm{C}$ e agitou-se vigorosamente por $1 \mathrm{minu}-$ to. Logo após foi deixado em repouso por 15 minutos.

A presença de saponinas é observada pela formação de espumas persistentes.

\section{RESULTADOS E DISCUSSÃo}

A triagem fitoquímica dos extratos etanólicos foliares de C. adamantium, D. mollis, $H$. stigonocarpa, K. lathrophytum e $S$. lycocarpum apresentaram positivo para ácidos orgânicos, açúcares redutores, alcaloides, catequinas, compostos cumarínicos, depsídeos, depsidonas, fenóis, flavonoides, glicosídeos cardiotônicos, saponinas e taninos. Foram observados testes negativos para polissacarídeos e purinas em todos os extratos avaliados conforme demonstrado na Tabela 1. 
Tabela 1. Perfil fitoquímico qualitativo dos extratos etanólicos foliares de Campomanesia adamantium, Dimorphandra mollis, Hymenaea stigonocarpa, Kielmeyera lathrophytum eSolanum lycocarpum.

Table 1. Qualitative phytochemical profile of leaf ethanolic extracts of Campomanesia adamantium, Dimorphandra mollis, Hymenaea stigonocarpa, Kielmeyera lathrophytum eSolanum lycocarpum.

\begin{tabular}{|c|c|}
\hline Classe dos metabólicos secundários & Resultados qualitativos \\
\hline Ácidos orgânicos & $(+)^{a}, b, c, d, e^{*}$ \\
\hline Açúcares redutores & $(+)^{a}, b, c, d, e^{*}$ \\
\hline Alcalóides & $(+) a, b, c, d, e^{*}$ \\
\hline Catequinas & $(+)$ a, c, d $(-)$ b, e* \\
\hline Cumarínicos & $(+)^{a}, \mathrm{~b}, \mathrm{c}, \mathrm{d}, \mathrm{e}^{*}$ \\
\hline Depsídeos e Depsidonas & $(+) a, b, c, d, e^{*}$ \\
\hline Duplas ligações olefínicas & $(-)$ a, b, c, d $(+) e^{*}$ \\
\hline Fenóis & $(+) a, b, c, d, e^{*}$ \\
\hline Flavonoides & $(+) \mathrm{a}, \mathrm{b}, \mathrm{d}, \mathrm{e}(-) \mathrm{c}^{*}$ \\
\hline Glicosídeos cardiotônicos: & \\
\hline Baljet & $(+) c, d(-) a, b, e^{*}$ \\
\hline Kedde & $(+)$ a, b, c, e $(-)^{d^{*}}$ \\
\hline Keller-Killiani & $(+) \mathrm{a}, \mathrm{b}, \mathrm{c}, \mathrm{e}(-) \mathrm{d}^{*}$ \\
\hline Raymond-Marthoud & $(+) \mathrm{a}, \mathrm{b}, \mathrm{c}, \mathrm{d}, \mathrm{e}^{*}$ \\
\hline Polissacarídeos & $(-)^{a}, b, c, d, e^{*}$ \\
\hline Purinas & $(-)$ a, b, c, d, e* \\
\hline Saponinas espumídicas & $(+) \mathrm{b}, \mathrm{c}, \mathrm{d}(-) \mathrm{a}, \mathrm{e}^{*}$ \\
\hline Taninos & $(\mathrm{Vd}) \mathrm{a}, \mathrm{b}, \mathrm{c}, \mathrm{d}, \mathrm{e}^{*}$ \\
\hline
\end{tabular}

De acordo com Brum et al. (2011), resultados negativos não implicam em sua ausência total destes compostos, possivelmente o nível mínimo de detecção estejam abaixo para os testes qualitativos avaliados. Os ácidos orgânicos neste estudo foram positivos para os extratos foliares etanólicos avaliados. Godinho et al. (2015)avaliando extratos E. dysenterica, A. fraxinifolium, $M$. urundewva $\mathrm{e} S$. lycocarpum encontraram a presença positiva corroborando com o resultado para S. lycocarpum neste estudo, e para B. gaudichaudii e $S$. paniculatumnão encontraram a presença para o extrato etanólico das folhas.

As plantas conseguem acumular quantidades expressivas de ácidos orgânicos no interior dos vacúolos, estes ácidos possuem ação bacteriostática em bactérias do grupo gramnegativa e são também utilizados em larga escala na indústria de alimentos como aditivos (MENEZES FILHO; CASTRO, 2019; GOMES; MARTINS; ALMEIDA, 2017).
Para o teste de açúcares redutores foram obtidos resultados positivos para todos os extratos foliares avaliados. Gomes, Martins e Almeida (2017) avaliando o perfil fitoquímico do extrato etanólico de Nephrolepis pectinata observou a presença positiva de açúcares redutores. Duarte, Mota e Almeida (2014) encontram açúcares redutores no extrato etanólico bruto das folhas de T. serratifolia. Os autores ainda complementam que a presença de açúcares redutores e não redutores possuem papel importante para aspecto de irradiação solar, onde plantas em pleno sol apresentam altos índices nos teores de açúcares.

Os principais açúcares redutores encontrados são, glicose e frutose, onde o primeiro atua no sistema nervoso central suprindo energia e previne contra a diabetes tipo 2 , e o segundo possui efeitos para reposição energética (GOMES; MARTINS; ALMEIDA, 2017; ARAÚJO; MARTEL, 2009; BARREIROS; BOSSOLAN; TRINDADE, 2005).

Os alcalóides foram positivos em todos os extratos etanólicos foliares avaliados neste estudo. Silva, Oliveira e Lima (2015) observaram a presença de alcalóides no extrato vegetal das folhas de S. terebinthifolius. Carrera et al. (2014) não encontraram reação positiva em extratos a quente e a frio em folhas de O. maculata. Brito et al. (2008) avaliando o extrato etanólico foliar de Annona squamosa obteve resultados também positivos. Os autores complementam que os alcalóides possuem ação biológica com açãoanti-hipertensiva, anti-inflamatória e antitumoral.

Para catequinas neste estudo, foram observados resultados positivos para C. adamantium, H. stigonocarpa, K. lathrophytum e negativo para D. mollis e S. lycocarpum. Souza et al. (2017) obtiveram na triagem fitoquímica para extratos hidroalcoólicos foliares de P. barbatus resultado positivo e para $L$. alba e P. anisumresultados negativos. Gomes, Martins e Almeida (2017) avaliando extrato foliar de Nephrolepis pectinata também observaram a presença de 
compostos catéquicos. Em estudos fitoquímicos Melo et al. (2004) observaram a presença de catequinas em extrato de Peumus boldus. A catequina tem como efeito a redução do tecido adiposo corporal baseado no metabolismo dos lipídeos e atividade antiulcerogênica (GOMES; MARTINS; ALMEIDA, 2017).

O teste para compostos cumarínicos foram positivos para todos os extratos etanólicos foliares avaliados neste estudo. Lima-Neto et al. (2015) avaliando extratos foliares de $L$. paniculata, H. speciosa, Q. grandiflora, K. coriaceae e $P$. rígida não apresentaram resultados positivos para compostos cumarínicos. Carrera et al. (2014) avaliaram os extratos obtidos a frio e a quente das folhas de O. maculata onde não foi observado a presença de compostos cumarínicos. Rodrigues, Souza-Filho e Ferreira (2009) avaliando o extrato bruto hidrometanólicos das folhas de Senna alata obtiveram resultado mascarado não sendo possível a constatação positiva ou negativa deste composto.

Às cumarinas estão envolvidas em uma grande variedade de atividades farmacológicas e bioquímicas as quais dependendo dos padrões de substituição; possuindo isômeros conhecidos por cromonas (5H-1-benzopiran5-onas) (KLOSS et al., 2016).

A avaliação qualitativa de depsídeos e depsidonas neste estudo apresentou em todas as amostras resultado positivo. Duarte, Mota e Almeida (2014) identificaram em teste qualitativo a presença de depsídeos e depsidonas em extrato bruto etanólico das folhas de T. serratifolia. Rodrigues, Souza-Filho e Ferreira (2009) obtiveram resultado mascarado para depsídios avaliando extrato hidrometanólicos das folhas de Senna alata. Macedo et al. (2007) detectaram a presença positiva de depsídeos e depsidonas no extrato foliar de $S$. adstrigens.

Os depsídeos e depsidonas fazem parte dos compostos fenólicos, ocorrendo em liquens, fungos e vegetais superiores. A rota biosintética para produção de xantonas acontece atra- vés da via do ácido chiquímico e ela via policetídica. As xantonas são precursoras de depsidonas em plantas superiores (gimnospermas e angiospermas) através da reação de hidroperoxilação, rearranjos de Baeryer-Villiger (DUARTE; MOTA; ALMEIDA, 2014).

De acordo com os autores Mota (2013), Micheletti et al. (2009) e Macedo et al. (2007), as depsidonas são um grupo de compostos estruturalmente relacionados aos depsídeos sendo estes, considerados seus precursores, com propriedades reconhecidas agindo como antioxidantes, antitumorais, analgésicas e antipiréticas.

Para o teste de duplas ligações olefínicas, apenas foi observado resultado positivo para o extrato etanólico deS. lycocarpum.CarvalhoJúnior et al. (2014) avaliando frações diclorometano do extrato foliar de E. copacabanensis encontraram carbonos olefínicos. As ligações duplas olefínicas podem ser definidas pela presença de dupla ligação entre átomos de carbonos em hidrocarbonetos (alcenos).

O nome olefina deriva do fato que hidrocarbonetos insaturados serem capazes de formarem compostos resinosos e oleosos, muitas das vezes pode apresentar aromas adocicados. As ligações duplas olefínicas podem estar associadas a reações de adição ou antioxidantes, dependendo da estrutura química na qual estão envolvidas.

Todos os extratos etanólicos foliares neste estudo apresentaram conteúdo qualitativo de fenóis. Souza et al. (2017) encontrou fenóis nos extratos hidroalcoólicos de $P$. barbatus e $P$. anisum através da triagem fotoquímica qualitativa. Gomes, Martins e Almeida (2017) obtiveram resultados positivos avaliando o extrato etanólico foliar de Nephrolepis pectinata. FélixSilva et al. (2012) encontraram fenóis em extratos aquosos de $A$. oleracea, $C$. ambrosioides, $L$. alba, M. piperita, O. gratissimum, P. boldus e R. officinalis.

De acordo com Menezes Filho e Castro (2019), e Duarte, Mota e Almeida (2014), os 
compostos fenólicos fazem parte de um grupo abundante bem diversificado apresentando estruturas simples e complexas e ao menos um anel aromático. Os compostos fenólicos contribuem no sabor, no odor e na composição de cores em diversos vegetais. Alguns compostos fenólicos tem apresentado ação antioxidante, sendo empregados na alimentação diminuindo a ação de reações oxidativas envolvidas em doenças como câncer e envelhecimento precoce.

Os flavonóides foram encontrados em $C$. adamantium, D. mollis, K. lathrophytum, S. lycocarpum não sendo observado em $H$. stigonocarpa. Gomes, Pena e Almeida (2016) obtiveram resultado positivo para flavonóides em extrato metanólico foliar de C. zeylanicum. Costa et al. (2014), observaram a presença de flavonóides em resíduo líquido de folhas de sisal com decocção e sem decocção. Farias, Costa e Takahashi (2011) caracterizando com diferentes solventes extratores sob o extrato de Banisteriopsis anisandra observaram resultados positivos para extrato foliar macerado com hexano, acetato de etila e por metanol.

Zuanazzi (2004) comenta sobre a importância dos flavonóides aos quais fazem parte de um dos grandes grupos de compostos fenólicos, de grande importância sendo encontrados nos mais variados tipos de vegetais sendo amplamente distribuída no grupo das angiospermas.

Para os testes de glicosídeos cardiotônicos neste estudo, foi observada para o reativo de Baljet a presença de resultados positivos para $H$. stigonocarpa, K. lathrophytum e negativo para C. adamantium, D. mollis, $H$. stigonocarpa e $S$. lycocarpum; para reação de Kedde apresentaram resultados positivos para C. adamantium, D. mollis e $H$. stigonocarpa; no reativo de KellerKilliani reação positiva para $C$. adamantium, D. mollis, $H$. stigonocarpae $S$. lycocarpum e negativo para $K$. lathrophytum e para o reativo de Raymond-Marthoud positivo para todos os extratos foliares.
Kloss et al. (2016) encontraram ausência de reação para o reativo de Kedde, KellerKilliani e Raymond-Marthoud, e reação positiva para o reativo de Baljet em extrato etanólico foliar de P. umbellatum. Análises fitoquímicas em extratos foliares de A.margravianum e A. nitidum realizadas por Añez (2009) revelaram a presença de glicosídeos cardiotônicos. Os compostos glicosídicos atuam no sistema cardíaco precisamente no miocárdio, sendo utilizado no tratamento de insuficiência cardíaca congestiva (KLOSS et al., 2016).

Não foram encontrados resultados positivos para a presença de polissacarídeos em nenhum dos extratos etanólicos foliares avaliados neste estudo. Gomes, Pena e Almeida (2016), analisando extratos hexânicos de acetato de etila e metanólico das folhas de $C$. zeylanicum, também não obtiveram resultados positivos para presença de compostos polissacarídicos.

Trindade et al. (2016) avaliando o extrato foliar de $A$. excelsum não detectou a presença de polissacarídeos avaliando dois testes fitoquímicos preliminares, para mucilagem (ácido tânico/cloreto férrico) e amido (lugol). Godinho et al. (2015) não encontrou compostos polissacarídicos em extratos de $B$. gaudichaudii, $E$. dysenterica, $A$. fraxinifolium, $M$. urundeuva, $S$. lycocarpum e $S$. paniculatum, e positivo para $A$. bumile. Bona et al. (2012) não encontram polissacarídeos no extrato hidroalcoólicos de $E$. mulungu.

Um dos principais polissacarídeos é o amido, utilizado na indústria de alimentos e como biomateriais sendo utilizado em processo cicatricial (SCHIRATO et al., 2006), os autores complementam sobre a utilização da goma extraída de $A$. occidentale que vem sendo utilizado apresentando resultados terapêuticos satisfatórios na cicatrização em lesões cutâneas em cobaias.

O teste para purinas neste estudo não constataram reações positivas em nenhum dos extratos foliares etanólicos avaliados. Resulta- 
dos iguais foram observados por Gomes, Martins e Almeida (2017) estudando o extrato bruto das folhas de Nephrolepis pectinata e Duarte, Mota e Almeida (2014) analisando o extrato foliar de Tabebuia serratifolia. Costa et al. (2009) avaliando extrato seco de alcachofra também não obteve resultado positivo para purinas.

As purinas são derivadas de aminoácidos como a glicina, ácido L-aspártico e Lglutamina. Essa classe de compostos orgânicos cíclicos do metabolismo secundário das plantas possui no mínimo um átomo de nitrogênio no seu anel sendo largamente utilizado na indústria farmacêutica como medicamentos alucinógenos e também como veneno (VIZZOTTO; KROLOW; WEBER, 2010).

Para saponinas neste estudo, houve a presença positiva para $D$. mollis, $H$. stigonocarpa e $K$. lathrophytum e negativo para C. adamantium e S. lycocarpum. Godinho et al. (2015) avaliou a formação de saponinas espumídicas em $S$. lycocarpum e obtiveram resultado negativo. Silva e Lima (2016) obtiveram resultados negativos para o extrato etanólico foliar de E. uniflora como também observados neste estudo para C. adamantium e S. lycocarpum. Silva, Oliveira e Lima (2015) identificaram no extrato etanólico das folhas de Schinus terebinthifoliusa presença de saponinas, neste estudo a presença de formação de espuma persistente foram positivas para D. mollis, $H$. stigonocarpa e $K$. lathrophytum.

Godinho et al (2015), e Schneider e Wolfling (2004), afirmam que as saponinas estão associadas as atividades hemolítica, antiinflamatória e na redução da falha congestiva cardíaca por inibição do sódio no fluxo celular.

Para taninos foram evidenciados resultados positivos para todos os extratos avaliados, aos quais apresentaram coloração verde na reação de identificação fitoquímica preliminar. Trindade et al. (2016) estudando o extrato foliar de Aspidosperma excelsum não encontraram a presença de compostos tanínicos. Já Costa et al. (2014) avaliando o resíduo líquido de sisal (Agave sisalana) com decocção e sem decocção obtiveram resultado positivo para taninos.

Carrera et al. (2014) observaram resultados positivos em extratos a frio e a quente de folhas de O. maculata. Mantovani e Porcu (2009) também encontraram a presença positiva para taninos totais e hidrolisáveis e ausência de reação positiva para taninos condensados em extrato aquoso foliar de Lippia alba.

De acordo com Kloss et al. (2016) os compostos tânicos são responsáveis pela percepção da adstringência pelo paladar visto em muitos frutos. Esta adstringência é devido à precipitação de compostos glicoproteicos salivares, perdendo o poder lubrificante. São compostos fenólicos altamente reagentes que formam ligações de hidrogênio intra e intermoleculares, sendo facilmente oxidáveis na presença de compostos enzimáticos e influência de metais (MONTEIRO; ALBUQUERQUE; ARAÚJO, 2005; MELLO; SANTOS, 2001).

Gomes, Martins e Almeida (2017), complementam sobre a ação terapêutica envolvendo compostos tânicos com ação antidiarreica e antisséptica por via interna e externa formando uma camada impermeabilizante as camadas da derme e de mucosas fazendo papel de proteção das camadas subjacentes.

De acordo com Miranda et al. (2016) e Matos (2009) os resultados preliminares da prospecção fitoquímica avaliando os metabólitos secundários constituintes de várias espécies vegetais podem diferenciar, isso se deve a fatores extrínsecos e intrínsecos como teores de minerais no solo, faixa de temperatura, água disponível e do próprio metabolismo secundário vegetal.

\section{CONSIDERAÇÕES FINAIS}

O trabalho comprovou cientificamente atividades farmacológicas identificadas através 
dos extratos etanólicos foliares e sua importância quanto à necessidade de se conhecer os princípios ativos contribuindo para o avanço das pesquisas científicas sobre compostos naturais na área de Fitoquímica. Os extratos etanólicos foliares apresentaram metabólitos secundários: ácidos orgânicos, alcaloides, glicosídeos cardiotônicos, flavonoides e triterpenos,

Os estudos desenvolvidos para se avaliarem os efeitos dos extratos possibilitam o conhecimento prévio, e indica a natureza química dos compostos naturais presentes nos preparados populares, e sua atuação em cosmetologia como cremes, pomadas de uso tópico e medicamentos de uso interno.

Por fim é necessário que as espécies vegetais do Cerrado avaliadas neste estudo, sejam submetidas a estudos fitoquímicos biomonitorados, com o objetivo de isolar cada classe de composto ativo, quantificando o seu teor e estabelecendo suas atividades com ação na produção de medicamentos ou mesmo compostos herbicidas naturais conciliando a preservação do Cerrado sendo alto sustentável economicamente.

\section{AGRADECIMENTOS}

Os autores agradecessem ao Instituto $\mathrm{Fe}$ deral Goiano - Campus Rio Verde; aos órgãos de fomento em pesquisa, CNPq, CAPES, FINEP e a FAPEG, esta última pela bolsa de mestrado em Agroquímica para o primeiro autor Antonio.

\section{REFERÊNCIAS}

AÑZZ, R. B. S. Análise morfoanatômica das folhas e casca de Aspidosperma nitidum Benth. eAspidosperma marcgravianum Woodson (Apocynaceae) com abordagem farmacognóstica e etnofarmacológica.2009. 126 f. Tese (Doutorado em Botânica), Instituto Nacional de Pesquisas da
Amazônia, Universidade Federal do Amazonas, UFAM, Manaus, 2009.

ARAÚJO, J. R.; MARTEL, F. Regulação da absorção intestinal de glicose. Arquivos de Medicina, Porto Mar, v. 23, n. 2, p. 35-43, 2009.

BARBOSA, W. R. L.; QUIGNARD, E.; TAVARES, I. C. C. et al. Manual para análise fitoquímica e cromatográfica de extratos vegetais. Revista Científica, v. 4, n. 5, p. 1-19, 2004

BARREIROS, C. R.; BOSSOLAN, G.; TRINDADE, P. E. C. Frutose em humanos: efeitos metabólitos, utilização clínica e erros inatos associados. Revista Nutrição, Campinas, v. 18, n. 3, p. 377-389, maio/jun., 2005. https://doi.org/10.1590/S1415-52732005000300010

BONA, A. P. de.; BATITUCCI, M. C. P.; ANDRADE, M. A. et al. Estudo fitoquímico e análise mutagênica das folhas e inflorescências de Erythrina mulungu (Mart. ex Benth.) através do teste de micronúcleo em roedores. Revista Brasileira de Plantas Medicinais, Botucatu, v. 14, n. 2, p. 344-351, 2012. https://doi.org/10.1590/S1516-05722012000200014

BRITO. H. O.; NORONHA, E. P.; FRANÇA, L. M. et al. Análise da composição fitoquímica do extrato etanólico das folhas da Annona squamosa (ATA). Revista Brasileira de Farmácia, v. 83, n. 3, p. 180-184, 2008.

BRUM, T. F. de et al. Análise fitoquímica preliminar das folhas de Vitex megapotamica (Sprengel) Moldenke. Revista Saúde, Santa Maria, v. 37, n. 2, jul./dez., 2011. https://doi.org/10.5902/223658343088

BUENO, M. L.; OLIVEIRA-FILHO, A. T. de.; PONTARA, V. et al. Flora arbórea do Cerrado de Mato Grosso do Sul. Revista Iheringia, Série Botânica, Porto Alegre, v. 73 (supl.), p. 53-64, mar., 2018. https://doi.org/10.21826/2446-8231201873s53

CARRERA, G. C. et al. Testes fitoquímicos em extratos foliares de Oeceoclades maculata Lindl. (Orchidaceae). Revista Brasileira de Plantas Medicinais, Campinas, v. 16, n. 4, p. 
938-944, 2014. https://doi.org/10.1590/1983$\underline{084 \mathrm{X} / 12174}$

CARVALHO JÚNIOR, A. R. de.; GOMES, G. A.; FERREIRA, R. O. et al. Constituintes químicos e atividade antioxidante de folhas e galhos de Eugenia copacabanensis Kiaersk (Myrtaceae). Revista Química Nova, v. 37, n. 3, p. 477-482, 2014.

COSTA, M. F.; OSUNA, J. T. A.; FRANDÃO, H. N. et al. Composição química e toxicidade foliar de extratos do resíduo líquido de sisal. Revista Magistra, Cruz das Almas, v. 26, n. 3, p. 372-384, jul./set., 2014.

COSTA, R. S.da.; OZELA, E. F.; BARBOSA, W. L. R.et al. Caracterização física, química e físico-química do extrato seco por nebulização (spray-drying) de Cynara scolmus L. (Asteraceae). Revista Brasileira de Farmacognosia, v. 90, n. 3, p. 169-174, 2009.

DUARTE, J. L.; MOTA, L. J. T.; ALMEIDA, S. S. M.da. S. de. Análise fitoquímica das folhas de Tabebuia serratifolia (Vahl.) Nicholson (Ipê Amarelo). Estação Científica (UNIFAP), Macapá, v. 4, n. 1, p. 33-43, jan./jun., 2014.

FARIAS, U. A. de.; COSTA, M. C. M.; TAKAHASHI, J. A. Caracterización fitoquímica y de las atividades antibacterianas y anticolinesterasa de Banisteriopsis anisandra A. Juss. (Malpighiaceae). Revista Cubana de Plantas Medicinales, Havana, v. 16, n. 1, p. 60-71, 2011.

FÉLIX-SILVA, J. et al. Identificação botânica e química de espécies vegetais de uso popular no Rio Grande do Norte, Brasil. Revista Brasileira de Plantas Medicinais, Botucatu, v. 14, n. 3, p. 548-555, 2012. https://doi.org/10. 1590/S1516-05722012000300018

GODINHO, C. S. et al. Estudo fitoquímico de espécies arbóreas do cerrado. Revista Multitexto, v. 3, n. 1, p. 64-70, fev., 2015.

GOMES, E. M. C.; PENA, R. da. C. M.; ALMEIDA, S. S. M. da. S. de. Composição fitoquímica e ação fungicida de extratos brutos de Cinnamomum zeylanicum sobre Quambalaria eucalypti. Biota Amazônia, Macapá, v. 6, n.
4, p. 54-58, 2016. https://doi.org/10.18561/21795746/biotaamazonia.v6n4p54-58

GOMES, N. M.; MARTINS, R. L.; ALMEIDA, S. S. M. da. S. de. Análise preliminar fitoquímica do extrato bruto das folhas de Nephrolepis pectinata. Estação Científica (UNIFAP), Macapá, v. 7, n. 1, p. 77-85, jan./abr., 2017. https://doi.org/10.18468/estcien.2017v7n1.p77-85

KLOSS, L. C.; ALBINO, A. M.; SOUZA, R. G. de. et al. Identificação de classes de metabólitos secundários do extrato etanólico de Piper umbellatum L. (PIPERACEAE). South American Journal of Basic Education, Technical and Technological, v. 3 n. 2, p. 118-128, 2016.

LIMA NETO, G. A.; KAFFASHI, S.; LUIZ, W. T. et al. Quantificação de metabólitos secundários e avaliação da atividade antimicrobiana e antioxidante de algumas plantas selecionadas do Cerrado de Mato Grosso. Revista Brasileira de Plantas Medicinais, v. 17, n. 4, supl. III, p. 1069-1077, 2015. https://doi.org/10.1590/1983-084x/14 161

LIMA, R. A.; SILVA, A. C. Identificação das classes de metabólitos secundários no extrato etanólico dos frutos e folhas de Eugenia uniflora L. Revista Eletrônica em Gestão, Educação e Tecnologia Ambiental, Santa Maria, v. 20, n. 1, p. 381-388, jan./abr., 2016.

LOLA-LUZ, T.; HENNEQUART, F.; GAFFNEY, M. Effect on yild, total phenolic, total flavonoid and total isothiocyanate content of two broccoli cultivars (Brassica oleracea var.italica) following the Application of a comercial brown seaweed extract (Ascopbyllum nodosum). Agricultural and Food Science, v. 23, n. 1, p. 28-37, feb., 2014. https://doi.org/ $10.23986 /$ afsci.8832

MACEDO, F. M. de.; MARTINS, G. T.; RODRIGUES, C. G. et al. Triagem fitoquímica do barbatimão [Stryphnodendron adstrigens (Mart) Coville]. Revista Brasileira de Biociências, Porto Alegre, v. 5, supl. 2, p. 1166 1168, jul., 2007.

MANTOVANI, D; PORCU, O. M. Avaliação fitoquímica do extrato de Lippia alba para 
utilização como antioxidante natural em alimentos. Revista Tecnológica, v. 18, p. 6974, 2009.

MATOS, F. J. A. Introdução a fitoquímica experimental. Fortaleza: Edições UFC. 2009. MELO, L. G de.; NASCIMENTO, V. T do.; AMORIM, E. L. C de. et al. Avaliação da qualidade de amostras comerciais de boldo (Peumus boldus Molina), pata-de-vaca (Baubinia spp.) e ginco (Ginkgo biloba L.). Revista Brasileira de Farmacognosia, v. 14, n. 2, p. 111-120, jul./dez., 2004. https://doi.org/10. 1590/S0102-695X2004000200004

MELLO, J. C. P.; SANTOS, S. C. Taninos. In: SIMÕES, C. M.; SCHENKEL, E. P.; GOSMANN, G. et al. Farmacognosia: da planta ao medicamento. Porto Alegre: UFRGS/UFSC, 2001. p. 517-543.

MENDONÇA, R. C.; FELFILI, J. M.; WALTER, B. M. T. et al. Flora vascular do cerrado: Checklist com 12.356 espécies. In: Cerra: ecologia e flora (SANO,S. M.; ALMEIDA, S. P.; RIBEIRO, J. F.). Planaltina, Embrapa, 2008. p. 417-1279.

MENEZES FILHO, A. C. P. de.; CASTRO, C. F. de. S. Análise fitoquímica preliminar de extratos foliares de orchidaceas (Cattleyawalkeriana Gardner), (OncidiumcebolletaSw.), (EncyclialinearifolioidesKraenzl.) e (Polystachya concreta (Jacq.) Garay\& H. R. Sweet). Revista Ensaios e Ciências: Biológicas, Agrárias e da Saúde, v. 23, n. 1, p. 16-23, 2019. https://doi.org/10.17921/1415-6938.2019v23n1p16$\underline{23}$

MENEZES FILHO, A. C. P. de.; CASTRO, C. F. de. S. Classes fitoquímicas de metabólitos secundários em extratos etanólicos foliares de espécies do Cerrado brasileiro. Revista Saúde \& Ciência Online, v. 8, n. 1, p. 45-61, jan./abr., 2019.

MICHELETTI, A. C.; BEATRIZ, A.; LIMA, D. P. de. et al. Constituintes químicos de Parmotrema lichexanthonicum Eliasaro \& Adler Isolamento, modificações estruturais e avaliação das atividades antibiótica e citotóxica. Revista Química Nova, v. 32, n. 1, p. 12-20,
2009. https://doi.org/10.1590/S0100-404220090001 $\underline{00003}$

MIRANDA, C. A. S. F.; CARDOSO, M. das. G.; BATISTA, L. R.et al. Óleos essenciais de folhas de diversas espécies: propriedades antioxidantes e antibacterianas no crescimento de espécies patogênicas. Revista Ciência Agronômica, Fortaleza, v. 47, n. 1, p. 213-220, jan./mar., 2016.

MONTEIRO, J. M.; ALBUQUERQUE, U. O.; ARAÚJO, E. L. Taninos: uma abordagem da química a ecologia. Revista Química Nova, v. 28, p. 892-896, 2005. https://doi.org/10.1590/S0100-40422005000500029

MOTA, L. J. T. Estudo químico e biológico das folhas e galhos de Hyptis crenata (Pohl.) ex Benth (Lamiaceae - Lamiles). 2013. 63 f. Dissertação (Mestrado em Ciência da Saúde). Programa de Pós-Graduação em Ciências da Saúde, Universidade Federal do Amapá, UFAP, Macapá. 2013.

MYRES, N.; MITTERMEIER, R. A.; MITTERMEIER, C. G. et al. Biodiversity hotspots for conservation priorities. Nature, v. 403, n. 6772, p. 853-858, fev.,2000. https://doi.org/10. $\underline{1038 / 35002501}$

RODRIGUES, I. M. C.; SOUZA-FILHO, A. P. S.; FERREIRA, F. A. Estudo fitoquímico de Senna alata por duas metodologias. Revista Planta Daninha, Viçosa, v. 7, n. 3, p. 507 513, 2009. https://doi.org/10.1590/S0100-83582 $\underline{009000300011}$

SCHIRATO, G. V.; MONTEIRO, F. M. F.; SILVA, F. de. O. et al. Polissacarídeo do Anacardium occidentale L. na fase inflamatória do processo cicatricial de lesões cutâneas. Ciência Rural, Santa Maria, v. 36, n. 1, p. 149-154, jan./fev., 2006. https://doi.org/10.1590/S0103$\underline{84782006000100022}$

SILVA, N. L. A. da.; MIRANDA, F. A. A.; CONCEIÇÃO, G. M. da. Triagem fitoquímica de plantas de Cerrado, da área de proteção ambiental municipal de Inhamum, Caxias, Maranhão. Scientia Plena, Sergipe, v. 6, n. 3, p. 1-17, 2010.

https://periodicos.unifap.br/index.php/estacao Macapá, v. 9, n. 1, p. 89-101, Jan./Mar. 2019 
SILVA, L. R. da. S.; OLIVEIRA, A. A. de.; LIMA, R. A. Identificação dos metabólitos secundários do extrato etanólico das folhas de Schinus terebinthifolius Raddi. South American Journal of Basic Education, Technical and Technological, v. 2, n. 2, p. 84-93, 2015.

SCHNEIDER, G.; WOLFLING, J. Synthetic cardenolides and related compounds. Current Organic Chemistry, Hungria, v. 8, n. 14, sep.,2004. https://doi.org/10.2174/1385272043369926

SOUZA, C. A. S.; ALMEIDA, L. N.; CRUZ, E. S. et al. Controle de qualidade físicoquímico e caracterização fitoquímica das principais plantas medicinais comercializadas na feira-livre de Lagarto-SE. Scientia Plena, Sergipe, v. 13, n. 9, p. 1-8, 2017. https://doi.org/10.14808/sci.plena.2017.094501

TRINDADE, R. C. dos. S.; KIKUCHI, T. Y. S.; SILVA, R. J. F. et al. Estudo farmacobotânico das folhas de Aspidosperma excelsum Benth. (Apocynaceae). Revista Fitos, Rio de Janeiro, v. 10, n. 3, p. 220-372, jul./set., 2016. https://doi.org/10.5935/2446-4775.20160019

DE UGAZ, O. L.; LOCK, O.Investigación Fitoquímica, Métodos em el estúdio de productos naturales. Peru: Universidad Pontifícia Catolica Del Peru, 1994.

VIZZOTTTO, M.; KROLOW, A. C.; WEBER, G. E. B. Metabólitos secundários encontrados em plantas e sua importância. Pelotas: Embrapa Clima Temperado, 2010. p.16.

ZUANAZZI, J. S. Flavonóides. In: SIMÕES, C. M. O. Farmacognosia da planta ao medicamento. Porto Alegre, Florianópolis: UFRGS/UFSCar, 2004. p. 742.

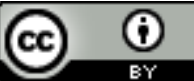

License information: This is an openaccess article distributed under the terms of the Creative Commons Attribution License, which permits unrestricted use, distribution, and reproduction in any medium, provided the original work is properly cited.

Artigo recebido em 14 de junho de 2018.

Avaliado em 05 de setembro de 2019.

Aceito em 06 de setembro de 2019.

Publicado em 02 de outubro de 2019.

Como citar este artigo (ABNT):

MENEZES FILHO, Antonio Carlos Pereira de; CASTRO, Carlos Frederico de Souza. Identificação das classes de metabólitos secundários em extratos etanólicos foliares de Campomanesia adamantium, Dimorphandra mollis, Hymenaea stigonocarpa, Kielmeyera lathrophytum e Solanum lycocarpum. Estação Científica (UNIFAP), Macapá, v. 9, n. 1, p. 89101, jan./mar. 2019. 